\title{
Management of Scars in Skin of Color
}

Huidi Tchero

\section{Contents}

43.1 Background -372

43.2 How Scars Evolve Differently in Skin of Color? - 372

43.3 Management of Scars in the Skin of Color - 373

43.3.1 Nonsurgical - 373

43.3.2 Surgical - 375

43.3.3 Response Rates and Side Effects in Skin of Color - 376

43.3.4 Recurrence Rate - 376

43.4 Management of Scars in Asian Skin - 376

43.5 Conclusions $-\mathbf{3 7 6}$

References -377 


\subsection{Background}

Scars are a normal process of dermal healing following laceration, skin incision, or tissue loss. Frequently, they are associated with burns, penetrating wounds, or surgical cuts. Morphologically, they appear erythematous and firm with elevated fibrous masses that are evolving within the margins of the wound and might revert later. They can cause cosmetic disfigurement, functional impairment, and psychological negative outcomes, affecting the patients' quality of life. These patients often seek medical advice to recover the color, texture, pliability, and relief of the associated pain [1]. In this chapter, we discussed how scars evolve differently in the skin of color, explored the available treatment modalities, and recommended planned treatment modalities in keloid management based on the published evidence.

\subsection{How Scars Evolve Differently in Skin of Color?}

Injury to the tissue could significantly produce inflammation, which is in turn associated with infiltration of neutrophils, monocytes, and macrophages, and release of pro-inflammatory cytokines, which induce fibroblast migration and proliferation, resulting in disproportionate extracellular matrix (ECM) deposition besides collagen accumulation [2]. This process is exaggerated when it affects the deep dermis, which would be complicated by the formation of a permanent scar. The scope of scar types varies from atrophic, flat, to raised dermal scars [3].

The pathogenesis of hypertrophic scars might be returned to atypical ECM metabolism following an odd and exaggerated fibroblastic stimulation. Histologically, it exhibits organized overexpression of types I and III collagen, deposition of fibronectin, and overexpression of pro-inflammatory interleukins (IL), such as IL-4, IL-6, IL-13, and IL-21, as well as downregulation of IL-12 and interferon (IFN)- $\gamma$ expression [2]. The incidence of hypertrophic scars associated with a burn is up to $78 \%$. The risk factor for hypertrophic scar includes dark skin, neck or upper limb age (younger), site, female gender, healing time, the severity of the injury, and the increase in a number of operations.

Keloids are different from hypertrophic scars in a variety of ways: they propagate outside the margins of the wound zone and invade the adjacent skin with no regression, conversely to hypertrophic scars that develop within the edges of the original wound and frequently regress in months. Keloids are a common fibroproliferative disorder that could happen due to abnormal healing process succeeding cutaneous injury. Keloids could evolve in any race; however, persons with more pigmented color (Fitzpatrick IV, V, and VI) are more vulner- able. In Hispanics, African-Americans, and Asians, keloids develop in up to $6-16 \%$ of those populations. The tendency for keloid expansion was also observed in patients suffering from dermatological diseases which lead to a protracted inflammatory reaction [1].

The presentation of keloid and hypertrophic scars can vary in patients with skin of color. Scars can be hyperpigmented or very large in patients with darker skin. Although keloid and hypertrophic scars appear usually within 1 year after trauma or surgery, few cases of people with skin of color who developed spontaneous keloid scars were reported within the literature as well.

Under the microscope, keloids appear as a twirling nodular arrangement of collagen fibers, characteristically with hyalinized, coarsened collagen bundles. Moreover, they have an acellular collagen center with adjoining hyper-proliferative fibroblasts. Their center covers dense fibers of immature collagen that are feebly supplied by blood vessels without lymphatic or elastin.

Several mechanisms have been hypothesized for the development of keloids, for example, deviant collagen production, abnormal growth factor regulation, and genetic vulnerability. Elevated levels of transforming growth factor beta (TGF- $\beta$ ) are suggested to be involved in keloid development; nonetheless, several other mediators are tending to be elaborated. Still, there is a lack of confirmed hypothesis that could elucidate keloid pathogenesis. Immune cells were also suggested to be involved in keloid scar formation.

Inherited genes increase predisposition for keloid tissue development; however, still, exact genes involved have not been acknowledged. Remarkably, keloid is exclusive to humans, and the animal model of keloid scars is absent. A large Japanese genome-wide association study (GWAS) discovered four single-nucleotide polymorphisms (SNPs) in three chromosomal segments in patients with keloid scars. These SNPs include rs8032158, situated in intron 5 of chromosome 15 that downregulates NEDD4 gene, which chiefly affects keloid severity. A study of admixture mapping recognized potentially mutual genetic regions between a group of Black, Chinese, and Japanese patients on chromosome 15q21.2-22.3, within which NEDD4 inhabits.

In analogous studies among relatives, one on chromosome $2 q 23$ and the other on chromosome $7 p 11$ were recognized in Japanese patients and African-American patients, respectively. Notwithstanding evidence of familial keloid formation, there was a changeability in phenotypic presentations and scar severity between families; hence, polygenic inheritance pattern is mostly included in keloid inheritance [4].

Although there are no clear evidence regarding the influence of inherited genes on the higher frequency or severity of scar among patients with skin of color, it was 
previously observed that the ethnicity of families with multiple affected members is mainly African-American and Indian. Variations in the age of onset, severity of the scars, and response to treatment in the same ethnic group may highlight the role of multiple gene mutations in the pathogenesis of the scar. Nevertheless, the pattern of gene involvements in scar pathogenesis is an area of further research.

\subsection{Management of Scars in the Skin of Color}

The prevention of scars can be achieved by proper cleaning and debridement of wounds, avoidance of unnecessary invasive procedures in high-risk population, and prophylactic measure. Modalities that prevent wound stretch - such as silicone gel sheets or tapes - and intralesional corticosteroids exhibited promising results inn scars prevention; however, the evidence is still weak.

To identify the most suitable management strategy, a thorough clinical examination should be done. This assessment must contain a full medical history, a detailed keloid scar history, any family history of keloids, and a psychosocial assessment. Moreover, a detailed examination should be conducted, including location, color, contour, size, pliability, and symptoms, i.e., pain and itching. Keloid is an untreatable disease, but nonetheless, physicians might be able to manage the manifestations of keloid, counting reducing scar size and redness and mitigating inflammation; this might reduce the recurrence rate of such lesions and may even yield complete extinction of lesions. Deterrence of signs and symptoms' exacerbation and impediment of recurrence after therapy are the main treatment goals of keloids.

Clinically, it is challenging to forecast if a scar will progress into either a hypertrophic scar or keloid. Increasing skin tension in specific locations and orientations while closing the wound and the existence of infections and foreign bodies may amplify the chance of keloid development. The more susceptible sites on the body for keloids include the earlobes, mandibular angle, posterior neck, shoulders, upper arms, upper back, and anterior chest. Bleeding, ulcerative, or firmly attached scars should undergo histopathologic investigation as a differential diagnosis of keloids. The possible differential lesions might be keloidal scleroderma, scar sarcoidosis, dermatofibroma, dermatofibrosarcoma protuberans, morphoeic basal cell carcinoma, and metastatic skin nodules.

Several therapeutic modalities are used in the management of keloid; though, due to unknown resolution rates and high recurrence rates, it is challenging to support any specific therapy. While several strategies exist, owing to the absence of level I evidence, none of them is universally demarcated as the standard. Presently, the treatments used have at best inadequate or indefinite effectiveness, with possible adverse drug reactions. Furthermore, many of these treatments require long treatment durations, might worsen the lesions, or could not prevent recurrence [3]. Multiple treatments for keloid, with varying efficacies, have been stated to date. Traditionally, a number of treatment regimens, for example, cryotherapy, interferon, and verapamil, have been applied for treating keloid, with unreliable success levels. Though, more novel treatments such as intralesional steroid injections, imiquimod, silicone gel, radiotherapy, lasers, electrical stimulation (ES), bleomycin, 5-fluorouracil (FU), surgery, and photodynamic therapy (PDT) have also been tested, not all have revealed efficacy in ethnic pigmented skin. These treatment modalities were reviewed in detail (• Fig. 43.1). However, due to the lack of studies on ethnic skin, some included studies presented data on pigmented, as well as nonpigmented keloid skin.

\subsubsection{Nonsurgical}

\subsubsection{Intralesional Steroid Injections}

Intralesional injection of corticosteroid (alone or with other agents) is one of the commonest treatment methods for hypertrophic scars and keloids. They are injected each 4-6 weeks as insoluble triamcinolone acetonide $(0-40 \mathrm{mg} / \mathrm{mL})$ until pain, scar, and pruritis subside. The response of patients to the treatment ranges from 50\% to $100 \%$, while the recurrence rate ranges from $9 \%$ to $50 \%$. However, 63\% presents with complications like localized ulceration, dermal atrophy, and telangiectasia or hypopigmentation. Noteworthy, pain might be managed through applying a local anesthetic. Corticosteroids alone represent the most efficient for young keloids, while older keloids are further resistant [2].

\subsubsection{Silicone Gel/Sheet}

Using silicone gel/sheets is assumed to diminish mobility and decrease the scar tension. How silicone gel works is uncertain; however, it may serve as an impermeable membrane to preserve hydration of the skin. A former RCT with 21 patients compared silicone gel with no therapy. Gold et al. found a reduction in hypertrophic scarring and keloid incidence following silicone gel use. However, the limitations of this trial were short followup period (only 12 weeks), and the blinding and techniques of randomization were not stated.

In another RCT by De Oliveira and colleagues, 26 patients suffering from keloids or hypertrophic scars had a mixture of silicone gel sheeting and non-silicone gel sheeting, while the control group received no treatment. They found no statistically significant difference 
- Fig. 43.1 Treatment modalities for a scar in colored skin

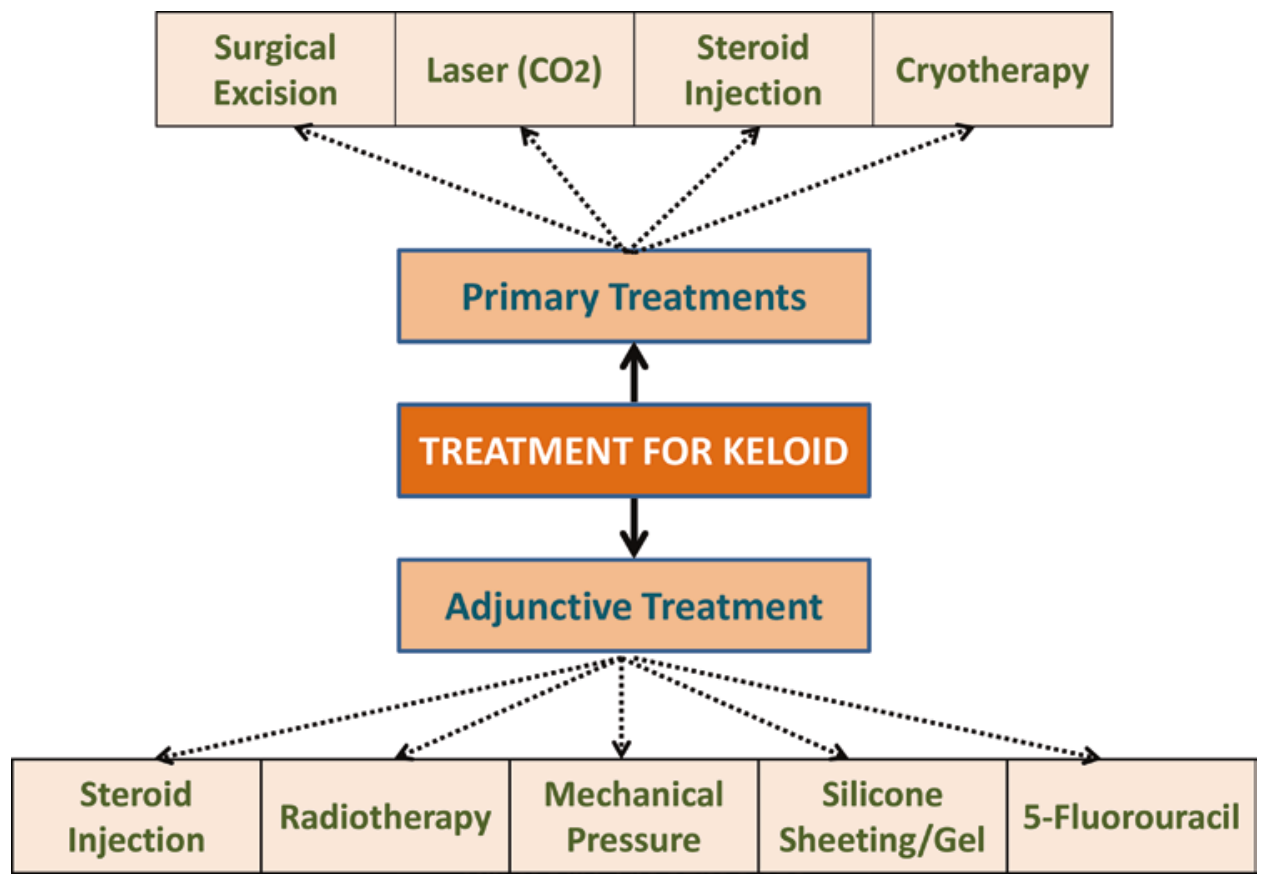

in the symptoms or size of scar by taking any of the treatments; however, both scar types were combined in their analysis, and the follow-up period was $<6$ months. These findings were in parallel with the results of O'Brien and Pandit study that was carried out among 13 trials including 559 subjects, observing the efficacy of silicone gel in the management of abnormal scarring in vulnerable groups. These studies collectively highlighted the demand for further controlled trials to approve the safety and efficacy of silicone gel sheeting.

\subsubsection{Radiotherapy}

Radiotherapy is preferred in elder adults who failed to respond to other treatments. It is used only in elderly patients because of the theoretical risk, however, there is a low risk of carcinogenesis. In resistant keloids, we could use ionizing radiation with intralesional excision. It acts via inhibiting collagen synthesis and inducing apoptosis of proliferating cells, which might rebuild a balance between keloid degeneration and collagen synthesis. It is given in doses of 15-30 Gy, ranging from 3 to 40 Gy among the studies, along six periods with accurate dosimetry and proper protection in the postoperative duration [2].

Doornbos et al. retrospectively analyzed 203 patients with keloid lesions using radiotherapy postsurgical excision. They elucidated the correlation between dose and response, with bigger doses decreasing the recurrence rate more efficiently. Conversely, Klumpar et al. found no significant dose-response correlation. Moreover, some investigators as Ragoowansi et al. and Maarouf et al. have supported the use of immediate postoperative radiotherapy [3]. Large-scale clinical trials are required to investigate a dose-response correlation among differ- ent races. Using radiotherapy as an adjuvant has revealed good scar resolution rates from $67 \%$ up to $98 \%$. Yet, the retrospective study design, different follow-up intervals, and lacking universal clinical evaluation have weakened this success rate.

\subsubsection{Photodynamic Therapy (PDT)}

The cytotoxic effects of PDT (with methyl-amino levulinate and amino levulinic acid) were investigated in different keloid lesional sites. One study concluded that the success of PDT depends on the photosensitizer precursor, the location, and the number of fibroblasts at the lesion site. A beneficial effect of topical application of methylamino levulinate PDT was initially observed in a patient with resistant keloid. There is a case series with 20 keloid patients who were examined for the effect of PDT. The results showed that PDT reduced pain and pruritus scores, reduced flow of blood, improved pliability and reduced levels of collagen in keloid, and resulted in a decrease of volume of keloid without recurrence over the follow-up period (9 months) [5]. These findings direct potential utility of PDT in the management of keloid and necessitate a further high-quality clinical trial to confirm the safety and efficacy of photodynamic therapy.

\subsubsection{Electrical Stimulation}

Electrical stimulation (ES) was investigated in relieving the keloid symptoms, such as pruritis and pain. Lately, a new in vitro system examined the efficacy of various ES types on collagen expression in keloid fibroblasts and revealed that ES could inhibit formation of collagen I in keloid. Two case series confirmed the efficacy of degraded wave ES in few patients with painful keloids, and the regimen exhibited significant amelio- 
ration of the symptoms [6]. Moreover, Sebastian and colleagues investigated the efficacy of PDT with and without ES and reported that the excitation improved the cytotoxic effects of PDT. Large-scale studies are needed to confirm the effectiveness of PDT-ES combination therapy.

\subsubsection{Surgical}

Surgery is needed in patients with hypertrophic scars with contractures, particularly when affecting joints that result in function loss. Hypertrophic scars may impede movement when they do abnormal forces on adjacent tissues or cross the joints. The elbow, knee, and shoulder are most commonly affected joints by contractures following burn injury. Surgery must be approached with caution as surgical scars may themselves increase the risk of keloid in pigmented skin. Recent studies showed no difference between surgical cutting modalities in terms of scar characteristics [7].

Surgical excision of keloids must be executed with substantial precaution, owing to the high recurrence risk. Excision could result in a bigger lesion and increased possibility of recurrence. The link between scarring and mechanical stress scarring is due to keloid scars occurring primarily in sites of excessive stress and mobility, for example, the shoulder, scapular region, and sternum. Therefore, it has been encouraged that cases subjected to surgery in these specific locations must have a long time of local skin support and splintage.

\subsubsection{Surgical Excision and Adjuvant Therapy}

The essential method in keloid treatment is surgical removal and primary closure, then injection of local steroid. Initial surgical excision followed by postoperative injection of steroid and silicone sheeting showed a $15 \%$ recurrence rate. Other modalities, for example, wearing a body corset or supportive bra, can furthermore decrease the risk of keloid in the mid-sternal region or upper trunk. Precautions to be considered while performing the first keloid surgery include not putting tension on the adjacent skin while closing the lesion. All possible causes of residual inflammation, i.e., entrapped hair follicles or dermal sinus tracts, should be excised to avoid recurrence. In case of large-sized keloid, serial excision would be well thought out to diminish skin tension. The injection of corticosteroid may be performed with surgery, then once every month for about 4-6 months. Surgeons should avoid local Z-plasties, $\mathrm{W}$-plasties, and skin flaps beyond the defect to avert recurrence of bigger keloid. Of note, the use of silicone as a prophylactic method has so far been recommended in many studies except for Niessen et al.'s study. Topical steroid tape has also exhibited effectiveness in prevent- ing recurrence, although it has the potential for unfortunate compliance because it is dependent on its continuous application by the patient [1].

\subsubsection{Cryosurgery}

Smaller keloid lesions have been treated by cryosurgery; however, substantial pain and occasionally prolonged healing times posttreatment limit its usage. The hypothesized mechanism of action is through ischemic damage that results in cellular anoxia producing necrosis of tissue. Furthermore, it modifies collagen synthesis and stimulates differentiation of keloid fibroblast near normal phenotype, thereby reducing keloid scar size [8].

Intralesional cryosurgery is a technique advanced from simple cryosurgery, which was first announced, by Shepard and Dawber. A simple cryosurgery, including pray technique or liquid nitrogen contact, etc., might produce vascular injury causing necrosis of tissue, anoxia, sloughing, and consequently scar flattening. This procedure could take two to ten treatment sessions with an interval of 20-30 days between each session. The success rate was reported ranging from 32\% to $74 \%$ following two or more therapies, with superior response rates among hypertrophic scars over keloids. There are complications such as pain and immediate blistering with long-term risk of dermal atrophy that could be hyper- or hypopigmented. Conversely, intralesional cryosurgery includes introducing a recent intralesional cryoneedle (Cryoshape ${ }^{\mathrm{TM}}$ ) inside the scar over the long axis. This probe is formed of an elongated uninsulated needle having double lumen with a safety vent and a sealed, cutting, distal tip prepared to augment the penetration of firm, dense scar. The probe proximal end is linked with liquid nitrogen that is pressurized to circulate within the needle that results in an ice ball forming around the cryoneedle leaving the adjoining scar tissue totally frozen, thus resulting in an apparently normal collagen regarding its structure and organization with a decrease in myofibroblasts and mast cells in the scar. This technique was first described in 1993 by Weshahy and later popularized by Har-Shai et al. and has revealed superior efficacy over simple cryosurgery, with a reported clinical effect ranging from $20 \%$ to $75 \%$ reduction in the scar volume. The main complications are peritreatment edema and epidermolysis, temporary hypopigmentation, and pain (while this pain is lower than simple cryotherapy). The main advantage of intralesional over simple cryotherapy was melanocyte sparing feature that accounts for a lower incidence of dyschromia as the temperature of skin surface is less influenced in intralesional cryotherapy [2].

A hospital-based clinical trial was performed on 30 patients with keloid using cryotherapy. The findings demonstrated that cryosurgery was an essential treatment regimen for recent keloid, chiefly in smaller lesions. Also, they found that both thicknesses and duration of 
keloid were the main agents in defining treatment outcome by cryosurgery. These findings were supported by Tziotzios and colleagues who recommended cryosurgery for reduction of scar size. Recently, a case study investigated the efficacy of cryosurgery plus surgical excision combination in 12 participants with keloid after 12 months. They demonstrated that shaving linked to cryosurgery was beneficial in the management of bulky keloid lesions because all cases showed improvement signs. Yet, it is arduous to follow this treatment modality owing to the small sample size [3].

\subsubsection{Response Rates and Side Effects in Skin of Color}

It is well-recognized that skin type significantly affects scar response to various modalities of treatment. Previous reports have shown that African-American patients had lower vascular response to topical corticosteroid than Caucasian patients. Relatively low response to bleomycin was reported among patients with skin of color. Patients with skin types IV-VI showed low response rates to different types of laser as well.

In terms of safety concerns, patients with skin of color are more reliable to higher incidence of adverse events than patients with lighter skin types. The risk of hypopigmentation after cryotherapy is more prominent in patients with darker skin due to cold sensitivity of melanocytes. While excessive melanin in patients with skin of color can absorb more laser and lead to hyperpigmentation, the high prevalence of melasma among patients with darker skin may contribute to post-laser hyperpigmentation as well. Hereditary hemolytic diseases are more prevalent among African-American, and they may impair healing after laser excision of the scars.

\subsubsection{Recurrence Rate}

Keloid scars are associated with high rate of recurrence, especially when treated with surgical excision alone. However, there are no current published data that address the effect of skin type on the rate of recurrence after scars treatment.

\subsection{Management of Scars in Asian Skin}

Recent guidelines on the management of scars in Asian patients recommended that scar prevention should be initiated in the immediate postoperative period owing to the high risk of developing poor scars. The recommended first-line therapy was silicone-based products (silicone gel and silicone gel sheets) based on their easy administration and strong supporting evidence. Several second-line therapies were recommended in case of failure of the first-line treatment, including intralesional steroid or 5-flourouracil injections, as well as radiotherapy. Despite lack of strong evidence, laser treatments have been increasingly accepted in these patients; however, further studies are needed to confirm its efficacy and determine the optimal wavelength and amount of energy required. Surgery is generally considered a last resort in resistant keloids. It can be combined with radiotherapy "sandwich technique," which has shown relatively low recurrence rates in Japanese patients [9].

\subsection{Conclusions}

Keloids are considered a challenging medical condition for both patients and medical health professionals owing to its high recurrence rate. The existing body of evidence has tried to investigate the pathophysiology and molecular basis involved in scar development using a reliable and reproducible model of a keloid. These endeavors would optimize the treatment and improve the outcomes of the disease. Currently, it is crucial to robust the published evidence, merged with clinical outcome, to deliver patients with the best therapeutic regimens for scars. Moreover, additional large-scale, long-term, and highquality RCTs are indispensable to conclude the safety and efficacy of these treatment options further, and also comparative studies are compulsory to identify the best treatment modality for scars in the pigmented skin. Finally, standard guidelines and protocols are mandatory to standardize the progress of such promising treatment modalities on a global scale.

The current body of evidence has emphasized the requirement for additional long-term clinical trials with larger sample size to assess the efficacy of the aforementioned treatments strongly. Several studies were short-term clinical trials with small numbers of patients included, and the others were case series. Besides, lacking stratification of patients into categories according to the type of lesion, i.e., keloid or hypertrophic scars, location, a degree of severity, or ethnicity of the patients, was present in some of these studies. The different types of scars are diverse at the molecular level and morphologically. Furthermore, the difference in skin color, site, number, and size of the scar could respond differently to the same modality of treatment. Therefore, this variability ought to be taken into consideration when designing those studies. Likewise, the measures used to assess scar characteristic and response to treatment were variable among the studies. Therefore, it is a compulsory requirement to use universal tool for 
assessment and implantation of measures to monitor cellular and metabolic activities in the tissue of keloid which, in turn, would support tailoring specific modality of treatment with each category of patients to ensure the best response of disease to treatment.

Keloid is unique for human tissue. This esoteric nature made keloids' preclinical studies scramble owing to the absence of animal models. Nonetheless, the advent of an in vitro organ culture model of keloid was fundamental in facilitating the study of disease molecular biology, pathophysiology, and the response among variable therapeutic modalities. This model was found to be a valuable tool for investigating new treatments and optimizing the treatment that would exhibit the optimum safety and efficacy in a diminution of the keloid scar mass size. Hence, it is considered as a prospective approach for new therapies assessment.

\section{Take-Home Messages}

- Pigmented skin has a higher chance of developing hypertrophic and keloid scars following wounds.

- Thorough history taking and clinical examination are essential for accurate assessment and management of abnormal scars in pigmented skin.

- Several therapeutic modalities for keloid management are available; however, there is lack of evidence to identify one strategy as the gold standard.

- Nonsurgical modalities for keloid management include intralesional steroid injections, radiotherapy, photodynamic therapy, and electrical stimulation.

- Surgery is needed in patients with hypertrophic scars with contractures, particularly when affecting joints that result in function loss.

- Combination therapies of surgical and nonsurgical modalities have shown some promising results; however, further research is needed.

- Large-scale clinical trials and clinical practice guidelines are needed to direct the development of effective treatments for keloid management.

\section{References}

1. Visscher MO, Bailey JK, Hom DB. Scar treatment variations by skin type. Facial Plast Surge Clin. 2014;22(3):453-62.

2. McGoldrick RB, Theodorakopoulou E, Azzopardi EA, Murison M. Lasers and ancillary treatments for scar management Part 2: keloid, hypertrophic, pigmented and acne scars. Scars Burns Heal. 2017;3:2059513116689805.

3. Ud-Din S, Bayat A. Strategic management of keloid disease in ethnic skin: a structured approach supported by the emerging literature. Br J Dermatol. 2013;169:71-81.

4. Andrews JP, Marttala J, Macarak E, Rosenbloom J, Uitto J. Keloids: the paradigm of skin fibrosis - pathomechanisms and treatment. Matrix Biol. 2016;51:37-46.

5. Ud-Din S, Thomas G, Morris J, Bayat A. Photodynamic therapy: an innovative approach to the treatment of keloid disease evaluated using subjective and objective non-invasive tools. Arch Dermatol Res. 2013;305(3):205-14.

6. Ud-Din S, Giddings PD, Colthurst J, Whiteside S, Morris J, Bayat A. Significant reduction of symptoms of scarring with electrical stimulation: evaluated with subjective and objective assessment tools in a prospective noncontrolled case series. Wounds. 2013;25(8):212-24.

7. Ismail A, Abushouk AI, Elmaraezy A, Menshawy A, Menshawy E, Ismail M, et al. Cutting electrocautery versus scalpel for surgical incisions: a systematic review and meta-analysis. J Surg Res. 2017;220:147-63.

8. Durani P, Bayat A. Levels of evidence for the treatment of keloid disease. J Plast Reconstr Aesthet Surg. 2008;61(1):4-17.

9. Kim S, Choi TH, Liu W, Ogawa R, Suh JS, Mustoe TA. Update on scar management: guidelines for treating Asian patients. Plast Reconstr Surg. 2013;132(6):1580-9.

Open Access This chapter is licensed under the terms of the Creative Commons Attribution 4.0 International License (http://creativecommons. org/licenses/by/4.0/), which permits use, sharing, adaptation, distribution and reproduction in any medium or format, as long as you give appropriate credit to the original author(s) and the source, provide a link to the Creative Commons license and indicate if changes were made.

The images or other third party material in this chapter are included in the chapter's Creative Commons license, unless indicated otherwise in a credit line to the material. If material is not included in the chapter's Creative Commons license and your intended use is not permitted by statutory regulation or exceeds the permitted use, you will need to obtain permission directly from the copyright holder.

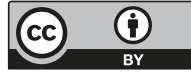

